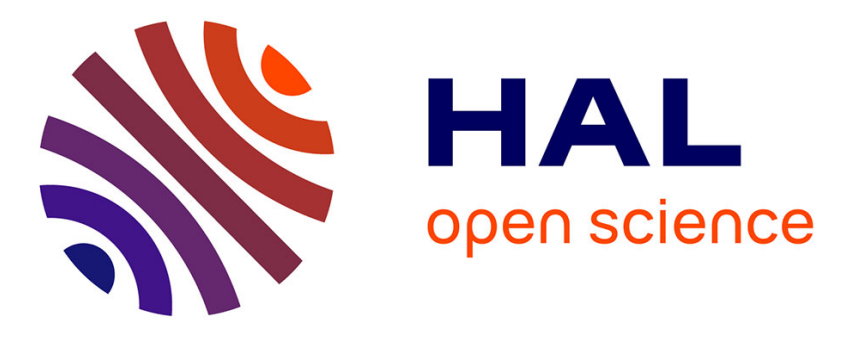

\title{
Correcting for Electrocatalyst Desorption and Inactivation in Chronoamperometry Experiments
}

Vincent Fourmond, Thomas Lautier, Carole Baffert, Fanny Leroux, Pierre-Pol Liebgott, Sebastien Dementin, Marc Rousset, Pascal Arnoux, David Pignol, Isabelle Meynial Salles, et al.

\section{To cite this version:}

Vincent Fourmond, Thomas Lautier, Carole Baffert, Fanny Leroux, Pierre-Pol Liebgott, et al.. Correcting for Electrocatalyst Desorption and Inactivation in Chronoamperometry Experiments. Analytical Chemistry, 2009, 81 (8), pp.2962 - 2968. 10.1021/ac8025702 . hal-01825488

\section{HAL Id: hal-01825488 \\ https://hal.science/hal-01825488}

Submitted on 31 May 2020

HAL is a multi-disciplinary open access archive for the deposit and dissemination of scientific research documents, whether they are published or not. The documents may come from teaching and research institutions in France or abroad, or from public or private research centers.
L'archive ouverte pluridisciplinaire HAL, est destinée au dépôt et à la diffusion de documents scientifiques de niveau recherche, publiés ou non, émanant des établissements d'enseignement et de recherche français ou étrangers, des laboratoires publics ou privés. 


\title{
Correcting for Electrocatalyst Desorption and Inactivation in Chronoamperometry Experiments
}

\author{
Vincent Fourmond, ${ }^{t, \neq}$ Thomas Lautier, ${ }^{\S, \|, \perp}$ Carole Baffert, ${ }^{t, \neq}$ Fanny Leroux, \\ Pierre-Pol Liebgott, ${ }^{\text {t, }}$ Sébastien Dementin, ${ }^{+, *}$ Marc Rousset, ${ }^{+, *}$ Pascal Arnoux, ${ }^{\ddagger, \#, \nabla}$ \\ David Pignol, ${ }^{\ddagger, \#, \nabla}$ Isabelle Meynial-Salles, ${ }^{\S, \|, \perp}$ Phillippe Soucaille, ${ }^{\S, \|, \perp}$ Patrick Bertrand, ${ }^{t, *}$ and \\ Christophe Léger*,t,*
}

Unité de Bioénergétique et Ingénierie des Protéines (BIP), IMM, UPR 9036, CNRS, 31 Chemin Joseph Aiguier, F-13402 Marseille Cedex 20, France, Aix-Marseille Université, 3 Place Victor Hugo, F-13333 Marseille Cedex 3, France, Université de Toulouse, INSA, UPS, INP, LISBP, 135 Avenue de Rangueil, F-31077 Toulouse, France, INRA, UMR792 Ingénierie des Systèmes Biologiques et des Procédés, F-31400 Toulouse, France, CNRS, UMR5504, F-31400 Toulouse, France, Laboratoire de Bioénergétique Cellulaire (LBC), SBVME, IBEB, CEA, F-13108 Saint-Paul-lez-Durance, France, and Laboratoire de Biologie Végétale et Microbiologie Environnementales (LBVME), UMR 6191, CNRS, F-13108 Saint-Paul-lez-Durance, France

Chronoamperometric experiments with adsorbed electrocatalysts are commonly performed either for analytical purposes or for studying the catalytic mechanism of a redox enzyme. In the context of amperometric sensors, the current may be recorded as a function of time while the analyte concentration is being increased to determine a linearity range. In mechanistic studies of redox enzymes, chronoamperometry proved powerful for untangling the effects of electrode potential and time, which are convoluted in cyclic voltammetric measurements, and for studying the energetics and kinetics of inhibition. In all such experiments, the fact that the catalyst's coverage and/or activity decreases over time distorts the data. This may hide meaningful features, introduce systematic errors, and limit the accuracy of the measurements. We propose a general and surprisingly simple method for correcting for electrocatalyst desorption and inactivation, which greatly increases the precision of chronoamperometric experiments. Rather than subtracting a baseline, this consists in dividing the current, either by a synthetic signal that is proportional to the instant electroactive coverage or by the signal recorded in a control experiment. In the latter, the change in current may result from film loss only or from film loss plus catalyst inactivation. We describe the different strategies for obtaining the control signal by analyzing various data recorded with adsorbed redox enzymes: nitrate reductase, NiFe hydrogenase, and FeFe hydrogenase. In each case we discuss the trustfulness and the benefit of the correction. This method also applies to experiments where electron trans-

* To whom correspondence should be addressed. E-mail: christophe.leger@ ibsm.cnrs-mrs.fr.

$\dagger$ BIP (CNRS)

* Aix-Marseille Université.

§ Université de Toulouse.

"INRA.

$\perp$ CNRS.

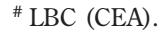

$\nabla$ LBVME (CNRS). fer is mediated, rather than direct, providing the current is proportional to the time-dependent concentration of catalyst.

Electrochemical experiments with adsorbed electrocatalysts are commonly used in analytical chemistry. Measuring a catalytic current as a function of time while the analyte concentration is being increased is a straightforward strategy for determining the linearity range of sensors and biosensors or the Michaelis constant for the substrate that is being transformed. For the last 15 years, chronoamperometric measurements with adsorbed redox enzymes have also been used for studying their kinetic properties. This made it possible to learn about many aspects of their mechanism that are otherwise difficult to investigate. ${ }^{1}$ One of the main advantages of this technique, compared to traditional solution assays with the enzyme in solution, is that, since the activity is measured as a current, it can be sampled at high frequency. This is useful for monitoring rapid changes in activity that result from the enzyme switching between catalytically active and inactive states under certain redox conditions or from the concentration of substrate or inhibitor being varied. ${ }^{1-5}$

In all cases, although the electrochemical nature of the measurement is ideally suited for quantitative measurements, a major experimental limitation is that the measured current is proportional to the catalyst's electroactive coverage; the latter may decrease over time, and the resulting change in current introduces systematic errors. This is a recurrent problem: browsing the last four issues of the journal Biosensors \& Bioelectronics (volume 24,

(1) Léger, C.; Bertrand, P. Chem. Rev. 2008, 108, 2379-2438, DOI: 10.1021/ cr0680742.

(2) Vincent, K. A.; Parkin, A.; Armstrong, F. A. Chem. Rev. 2007, 107, 4366, DOI: 10.1021/cr050191u.

(3) Léger, C.; Dementin, S.; Bertrand, P.; Rousset, M.; Guigliarelli, B. I. Am. Chem. Soc. 2004, 126, 12162, DOI: 10.1021/ja046548d.

(4) Almeida, M. G.; Guigliarelli, B.; Bertrand, P.; Moura, J. J. G.; Moura, I.; Léger, C. FEBS Lett. 2007, 581, 284, DOI: 10.1016/j.febslet.2006.12.023.

(5) Leroux, F.; Dementin, S.; Burlat, B.; Cournac, L.; Volbeda, A.; Champ, S.; Martin, L.; Guigliarelli, B.; Bertrand, P.; Fontecilla-Camps, J.; Rousset, M.; Léger, C. Proc. Natl. Acad. Sci. U.S.A. 2008, 105, 11188-11193, DOI: 10.1073/pnas.0803689105. 
issues $1-4)$, we found 29 articles showing chronoamperograms, out of which 13 were distorted by the desorption and/or inactivation of the electrocatalyst. ${ }^{6}$ The extent to which this actually limits the information that is gained varies greatly, but the error introduced by this effect is difficult to evaluate, because it cannot be decreased by statistical means, e.g., by repeating measurements and averaging large numbers of results. Regarding redox enzymes noncovalently adsorbed onto electrodes, film stability ranges from tens of hours or more $\mathrm{e}^{20,21}$ to only minutes in the worst cases, ${ }^{22,23}$ and the mechanistic information must be gained while the film is falling off. Chronoamperometry at the rotating disk electrode is often used for determining a Michaelis constant, but desorption precludes any accurate measurement of this parameter. ${ }^{24}$ Most problematically, film loss may also hide small changes in activity whose detection might otherwise have provided useful information, as illustrated hereinafter.

Here we present a very simple and powerful strategy for dealing with the systematic errors introduced by film loss and for correcting the data in an objective manner. This may not stabilize the biosensor or the film of enzyme, but their characterization will be greatly facilitated. This method is applicable if the current is the product of the time-dependent electroactive coverage $g(t)$, whose influence we seek to remove, times the rate of catalysis $k(t)$, which is the signal we are ultimately interested in. Both contributions (coverage and activity) can depend on time, electrode potential, and substrate or inhibitor concentration.

$$
i(t)=g(t) k(t) \quad \text { (principal signal) }
$$

The method we present is based on the possibility to either record independently or synthesize a control signal, $i^{\prime}(t)$, which is proportional to the time-dependent electroactive coverage during the principal experiment:

$$
i^{\prime}(t)=\alpha g(t) \quad \text { (control signal) }
$$

Dividing $i(t)$ by the control signal $i^{\prime}(t)$ yields the signal of interest, $k(t)$, up to a factor of proportionality $1 / \alpha$. That this factor is unknown will not preclude the analysis of the dependence of $k$ on time or substrate/inhibitor concentration. Indeed, in voltammetric experiments with adsorbed catalysts, particularly with enzymes, the electroactive coverage is often unknown, and the information is usually gained from relative changes in current, i.e., from the shape of the electrochemical signal, rather than from its absolute magnitude.

In more complex situations, the change in activity that is of interest is superimposed on a background decrease in current

(6) See Figures 5A, 3, 3A, 5B, 5A, 4, 5A, 3A, 3, 5A, 3B, 5, and 4 in refs $7-19$, respectively.

(7) Guascito, M.; Filippo, E.; Malitesta, C.; Manno, D.; Serra, A.; Turco, A. Biosens. Bioelectron. 2008, 24, 1063-1069, DOI: 10.1016/j.bios.2008.07.048.

(8) Ai, H.; Huang, X.; Zhu, Z.; Liu, J.; Chi, Q.; Li, Y.; Li, Z.; Ji, X. Biosens. Bioelectron. 2008, 24, 1054-1058, DOI: 10.1016/j.bios.2008.07.039.

(9) Deng, L.; Wang, Y.; Shang, L.; Wen, D.; Wang, F.; Dong, S. Biosens. Bioelectron. 2008, 24, 957-963, DOI: 10.1016/j.bios.2008.07.066.

(10) Guo, C.; Hu, F.; Li, C.; Shen, P. Biosens. Bioelectron. 2008, 24, 825-830, DOI: $10.1016 /$ j.bios.2008.07.007.

(11) Salimi, A.; Noorbakhash, A.; Sharifi, E.; Semnani, A. Biosens. Bioelectron. 2008, 24, 798-804, DOI: 10.1016/j.bios.2008.06.046.

(12) Wu, L.; Lei, J.; Zhang, X.; Ju, H. Biosens. Bioelectron. 2008, 24, 644-649, DOI: $10.1016 /$ j.bios.2008.06.009.

(13) Kafi, A.; Wu, G.; Chen, A. Biosens. Bioelectron. 2008, 24, 566-571, DOI: 10.1016/j.bios.2008.06.004. that is caused by an unavoidable inactivation of the electrocatalyst, in addition to film loss. In that case, $g(t)$ represents the amount of electrocatalyst that has neither desorbed nor inactivated by this process, and the correction eliminates the effects of both desorption and inactivation, as illustrated hereinafter.

In this paper, we use various experimental data to illustrate the distinct strategies that can be used for obtaining the control signal. In each case we demonstrate that the correction greatly enhances the information that is gained from the experiment. We have implemented the corresponding procedures in a free dataanalysis software.

\section{RESULTS AND DISCUSSION}

No Need to Record a Control Signal When the Change in Current Is a Step Function Distorted by Film Loss. The red trace in Figure 1B reminds the chronoamperograms reported in many studies of amperometric sensors ${ }^{7-19}$ or enzymes (see, e.g., Figure 5 in ref 23 or Figure 2A in ref 25). This signal was obtained with Rhodobacter sphaeroides $\mathrm{NapAB}$, a dimeric enzyme that catalyzes the two-electron reduction of nitrate to nitrite. ${ }^{26}$ The enzyme was adsorbed on a graphite electrode, ${ }^{27-29}$ which was rotated at high speed to avoid any limitation by mass transport and poised at low potential. The negative current, which is proportional to the rate of catalytic nitrate reduction, was monitored while nitrate concentration was increased by repeatedly injecting aliquots of a concentrated stock solution of $\mathrm{NO}_{3}{ }^{-}$in the electrochemical cell (Figure 1A). The turnover rate is constant between any two successive injections because catalysis is in the steady state; therefore, the decrease in the magnitude of the current that is observed on certain intervals (Figure 2A) is a

(14) Vazdominguez, C.; Campuzano, S.; Rudiger, O.; Pita, M.; Gorbacheva, M.; Shleev, S.; Fernandez, V.; Delacey, A. Biosens. Bioelectron. 2008, 24, 531537, DOI: 10.1016/j.bios.2008.05.002.

(15) Ozcan, L.; Sahin, Y.; Turk, H. Biosens. Bioelectron. 2008, 24, 512-517, DOI: 10.1016/j.bios.2008.05.004

(16) Zhou, M. Biosens. Bioelectron. 2008, 24, 442-447, DOI: 10.1016/ j.bios.2008.04.025.

(17) Du, F. Biosens. Bioelectron. 2008, 24, 415-421, DOI: 10.1016/ j.bios.2008.04.020.

(18) Cao, Z.; Jiang, X.; Xie, Q.; Yao, S. Biosens. Bioelectron. 2008, 24, 222-227, DOI: 10.1016/j.bios.2008.03.021.

(19) Dondapati, S.; Lozanosanchez, P.; Katakis, I. Biosens. Bioelectron. 2008, 24, 55-59, DOI: 10.1016/j.bios.2008.03.022.

(20) Blanford, C. F.; Heath, R. S.; Armstrong, F. A. Chem. Commun. 2007, 1710, DOI: $10.1039 / \mathrm{b} 703114 \mathrm{a}$.

(21) Blanford, C. F.; Foster, C. E.; Heath, R. S.; Armstrong, F. A. Faraday Discuss. 2009, 140, 319-335, DOI: 10.1039/b808939f.

(22) Heffron, K.; Léger, C.; Rothery, R. A.; Weiner, J. H.; Armstrong, F. A. Biochemistry 2001, 40, 3117, DOI: 10.1021/bi002452u.

(23) Barker, C. D.; Reda, T.; Hirst, J. Biochemistry 2007, 46, 3454, DOI: 10.1021/ bi061988y.

(24) Film loss while the substrate concentration is being increased leads to underestimating the current at high substrate concentration and, therefore, the value of $K_{\mathrm{m}}$; see the discussion in section 3.1.4 of ref 1 .

(25) Wijma, H. J.; Jeuken, L. J. C.; Verbeet, M. P.; Armstrong, F. A.; Canters, G. W. I. Biol. Chem. 2006, 281, $16340-16346$, DOI: 10.1074/ jbc.M601610200.

(26) Arnoux, P.; Sabaty, M.; Alric, J.; Frangioni, B.; Guigliarelli, B.; Adriano, J. M.; Pignol, D. Nat. Struct. Mol. Biol. 2003, 10, 928, DOI: 10.1038/nsb994.

(27) Frangioni, B.; Arnoux, P.; Sabaty, M.; Pignol, D.; Bertrand, P.; Guigliarelli, B.; Léger, C. J. Am. Chem. Soc. 2004, 126, 1328, DOI: 10.1021/ja0384072.

(28) Bertrand, P.; Frangioni, B.; Dementin, S.; Sabaty, M.; Arnoux, P.; Guigliarelli, B.; Pignol, D.; Léger, C. I. Phys. Chem. B 2007, 111, 10300, DOI: 10.1021/ jp074340j.

(29) Fourmond, V.; Burlat, B.; Dementin, S.; Arnoux, P.; Sabaty, M.; Boiry, S.; Guigliarelli, B.; Bertrand, P.; Pignol, D.; Léger, C. J. Phys. Chem. B 2008, 112, 15478, DOI: 10.1021/jp807092y. 


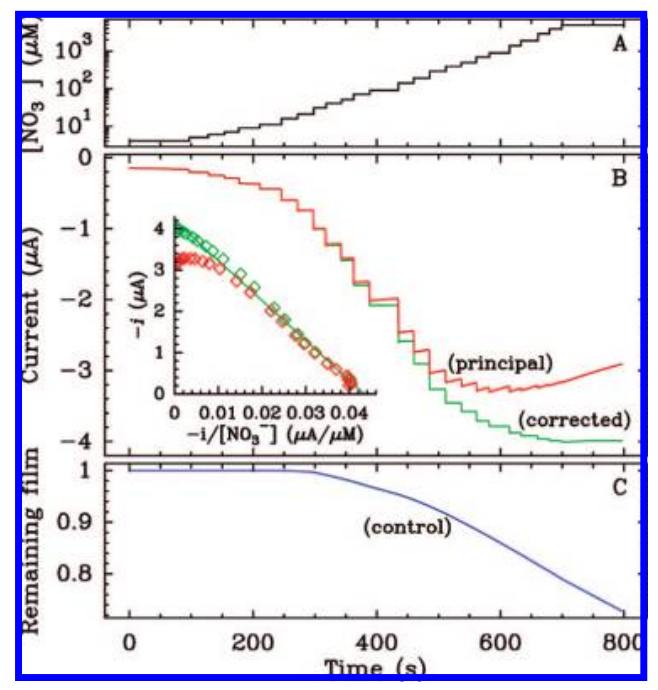

Figure 1. (A) Nitrate concentration against time. (B) Raw and corrected chronoamperograms (red and green lines) for $R$. sphaeroides nitrate reductase (NapAB) adsorbed at a rotating electrode. The current is proportional to the rate of nitrate reduction. Each increase in current results from nitrate being added; $\mathrm{pH} 7, T=25^{\circ} \mathrm{C}, \omega=5$ $\mathrm{krpm}, E=-510 \mathrm{mV}$ vs SHE. The inset shows the Eadie-Hofstee plots of rate against rate divided by substrate concentration. Such plot is linear if the data follow Michaelis-Menten kinetics (ref 30). (C) Normalized electroactive coverage reconstituted piece by piece from the data in panel B and used as a synthetic control signal for correcting the raw chronoamperogram.

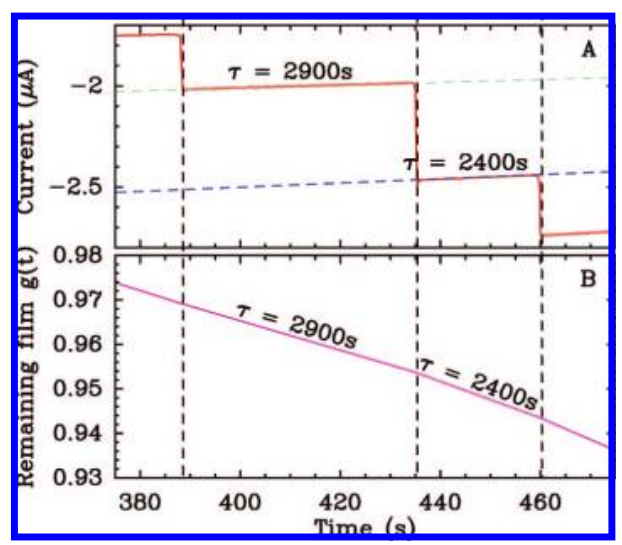

Figure 2. Synthesis of the control signal shown in Figure 1C. Panel $\mathrm{A}$ is a close-up on the raw data in Figure $1 \mathrm{~B}$, illustrating the piece by piece construction of the control signal shown in Figure 1C. $\tau$ is the time constant of film loss on each interval.

consequence of film desorption. The data in Figure 1 show that the film is all the less stable that substrate concentration is large.

Film loss makes it difficult to estimate the value of $K_{\mathrm{m}}$ : fitting to the Michaelis-Menten equation the uncorrected data obtained by taking current values at the beginning of each interval returns a $K_{\mathrm{m}}$ of about $60 \mu \mathrm{M}$, but the Eadie-Hofstee plot in the inset strongly deviates from linearity. Clearly, the decrease in current at high concentration is artifactual and leads to underestimating both the maximal current (at infinite concentration of nitrate) and the Michaelis constant.

We will show that, in this situation, it is possible to directly infer the time dependence of the electroactive coverage from the

(30) Cornish-Bowden, A. Fundamental of Enzyme Kinetics; Portland Press: London, 2004. measured current. Film loss should lead to an exponential decay of the current, and the observation that it decreases linearly with time (Figure 2A) shows that the time constant of the decay is much greater than the interval between two injections. Therefore, it is possible to independently fit each segment to a line of equation $y=a t+b$ which approximates the exponential decay whose time constant is $\tau=-\left(a t_{0}+b\right) / a$, where $t_{0}$ is the abscissa of the first point in the interval, and we build $g(t)$ piece by piece by using the value of $\tau$ deduced from the fit of each segment of the principal signal. We construct the normalized electroactive coverage $g(t)$ according to the following rules: (i) $g\left(t_{0}^{+}\right)=g\left(t_{0}^{-}\right)$, that is, there is no instant loss of electroactive coverage upon addition of substrate. (ii) $g(t)=g\left(t_{0}^{+}\right)(a t+b) /\left(a t_{0}+b\right)$ during the interval (Figure 2B). Figure $1 \mathrm{C}$ shows the entire function $g(t)$ calculated using the initial value $g(0)=1$. Dividing the original signal $i(t)$ by the reconstituted control signal yields the green step function in Figure 1B, where film desorption is no longer apparent. The Eadie-Hofstee plot shows that the corrected data do not deviate from the expected Michaelian behavior. The slope of this Eadie-Hofstee plot is $K_{\mathrm{m}}=97 \pm 3 \mu \mathrm{M} \cdot{ }^{31}$ Independent runs gave the same value within $5 \mu \mathrm{M}$.

This procedure can be applied whenever the function $k(t)$, i.e., the activity of the enzyme or the response of the sensor, is a step function. It may be used for precisely determining either Michaelis or inhibition constants, in experiments where the substrate or inhibitor concentration is stepwise increased. It should increase the accuracy of amperometric sensors. We have implemented the above correction procedure in a software we freely distribute; with the use of this program, constructing the $g(t)$ function and correcting the data according to the above method is only a matter of minutes (see the Methods section).

Control Experiment Carried Out with the Same Film When the Catalyst Is Irreversibly Transformed by the Reaction of Interest. When $k(t)$ is not a step function, the principal signal must be divided by a control signal in which the decrease in current reveals only the effects that should be corrected for, e.g., film loss. If the process whose kinetics one seeks to define occurs spontaneously, but only once per film, the control signal can be obtained from a subsequent experiment carried out with the same film. We used this strategy in our recent study of the irreversible activation of $R$. sphaeroides nitrate reductase, taking advantage of the fact that this activation proceeds only the first time the enzyme is reduced. ${ }^{29}$

$R$. sphaeroides nitrate reductase, the enzyme used in the experiment described in Figure 1, can be purified as either a dimer (NapAB) or a monomer (NapA). ${ }^{26}$ The films of the dimeric form are much more stable than those of the monomer. This is most likely because $\mathrm{NapB}$ is the proteic subunit that helps NapAB interact with the electrode. The enzyme undergoes an irreversible first-order activation the first time it is taken to low potentials $\left(E<-250 \mathrm{mV}\right.$ vs SHE).$^{29}$ In the case of NapAB, this is clearly seen as an increase over time of the nitrate reduction current (see ref 29 and Supporting Information Figure S1), but the analysis of the activation process of NapA is difficult because, in addition to electroactive coverage being smaller, film loss tends to make the current decrease faster than the enzyme activates. This is

(31) This error is the $95 \%$ confidence interval calculated from the covariance matrix. For details, see, e.g., section 4.B in the manual of the software ODRPACK at http://www.netlib.org/odrpack/. 


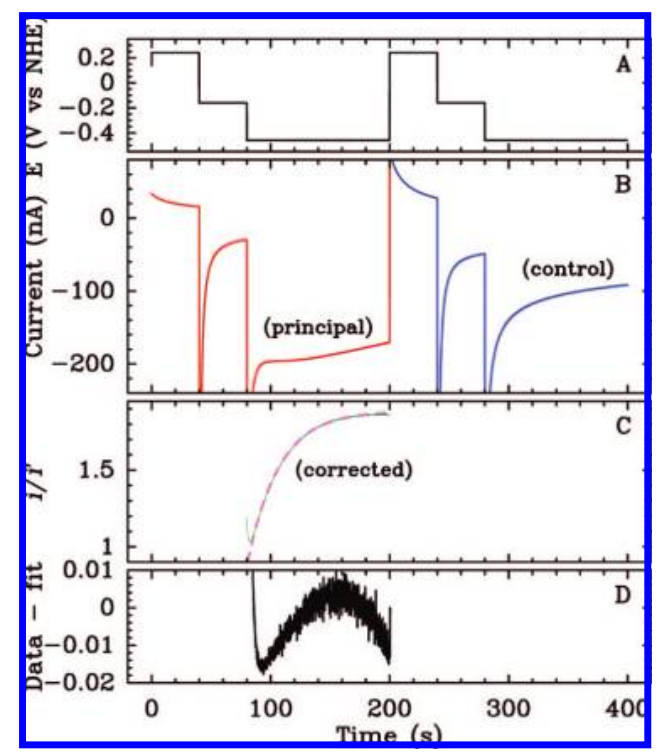

Figure 3. Chronoamperograms demonstrating the irreversible reductive activation of nitrate reductase. Panel A shows the sequence of potential steps at $+240,-160$, and $-460 \mathrm{mV}$, which was applied to a fresh film of as-prepared $R$. sphaeroides NapA. Panel B shows the principal experiment and the subsequent control experiment (red and blue traces, respectively). (C) Corrected data (green) and their fit to $y(t)=y_{0}\left(1-\beta \exp \left(-k_{\mathrm{a}} t\right)\right)$ (dashed magenta). Panel D shows the difference between the corrected data and the fit; $\mathrm{pH} 7$, $T=25^{\circ} \mathrm{C}, \omega=5 \mathrm{krpm},\left[\mathrm{NO}_{3}{ }^{-}\right]=100 \mu \mathrm{M}$.

illustrated by the red chronoamperogram in Figure 3: panel A shows the sequence of electrode potential steps which was applied to a fresh film on NapA. Panel B shows that the nitrate reduction current evolves in a complex manner, but clearly, at a given potential, the magnitude of the current continuously decreases over time. (The sharp variations of current result from double layer effects when the potential is stepped.) The control signal recorded with the same film, under the same conditions but after the enzyme has fully activated, is shown as a blue trace in panel B. The ratio of the two is shown in green in Figure 3C.

The corrected signal in Figure $3 \mathrm{C}$ is the change in activity induced by the first reduction of the enzyme. The fit of the activation phase to $y(t)=y_{0}\left(1-\beta \exp \left(-k_{a} t\right)\right)$ is perfect (dashed purple line); this demonstrates that the activation simply follows first-order kinetics and that the correction was successful. The fit returns the first-order rate of activation $\left(k_{\mathrm{a}}\right)$ and the relative amplitude of the activation $(\beta)$. In ref 29 , we show that the latter equates the fraction of enzyme that was initially inactive. We deduced $k_{\mathrm{a}}=31 \times 10^{-3} \mathrm{~s}^{-1}$ and $\beta=0.6$ from the data in Figure 3 , which is consistent with our previous results. ${ }^{29}$ Ten independent experiments returned values of $\beta$ in the range of $0.6-0.7$, and the dispersion of the values of $k_{\mathrm{a}}$ was $\pm 10 \%$.

We conclude that the corrected data clearly show that NapA activates under reducing conditions, and the kinetics of this process is easily characterized, despite the fact that the film is very unstable and activation is hidden by film loss in the raw signal. That the same activation process is observed with both NapA and NapAB clearly demonstrates that it results from a chemical transformation of the subunit NapA. ${ }^{29}$

Control Experiment Carried Out with a Fresh Film When the Reaction of Interest Is Triggered by the User. The use of two different films for the principal and the control experiments should prove useful if (i) the process of interest is triggered by the user during the principal experiment and (ii) the background decrease in current is not an exponential decay toward zero (if it is, there is no need to use a different film). Both conditions are fulfilled, for example, in experiments with hydrogenases adsorbed onto electrodes poised at very high potential, under conditions commonly used for studying the oxygen sensitivity of these enzymes. ${ }^{1,2}$

To characterize the kinetics of inhibition of hydrogenases by $\mathrm{O}_{2}$ using direct electrochemistry, a method consists in injecting in the electrochemical cell an aliquot of aerated solution while the potential of the enzyme-coated electrode is poised at a high value and the $\mathrm{H}_{2}$-oxidation activity is continuously monitored. ${ }^{3-5,32}$ Oxygen concentration increases within a fraction of a second after the injection, and then slowly returns to zero because hydrogen is flowed through the buffer. The whole process takes a few minutes, during which the activity is continuously measured, and the data can be analyzed without having to independently measure the time-dependent concentration of $\mathrm{O}_{2}$ (see section 2.4.2 in ref 1). Such experiments easily yield inhibition constants; ${ }^{3,33}$ most importantly, any delay in inhibitor binding and release can be interpreted to determine the rates of inhibition. ${ }^{4,5,32}$

This method can be used with any gaseous inhibitor, but when it is molecular oxygen, the electrode potential must be high enough that it is not directly reduced onto the electrode, else this would add a faradaic contribution to the current and decrease the effective oxygen concentration in the vicinity of the electrode. However, under such very oxidizing conditions, hydrogenases slowly inactivate even before oxygen is added, because the active site is slowly oxidized into one of its inactive forms (the so-called $\mathrm{NiB}$ and $\mathrm{H}_{\text {inact }}^{\mathrm{ox}}$ states of $\mathrm{NiFe}$ and $\mathrm{FeFe}$ hydrogenases, respectively). ${ }^{34}$ Anaerobic inactivation and film loss combine to produce a decrease in hydrogen oxidation current that appears to be at least biphasic, ${ }^{35,36}$ although the reason for this has not been clarified yet. The dashed line in Figure 4B shows such current decay recorded with Clostridium acetobutylicum $\mathrm{FeFe}$ hydrogenase adsorbed at a rotating disk graphite electrode ${ }^{32}$ The semilog plot in the inset clearly shows the deviation from firstorder kinetics. The effect of adding oxygen in the buffer superimposes on this complex decay to produce the signal shown as a plain line in Figure 4B, where the three abrupt variations in current result from injections of aliquots of solution equilibrated under 1 atm of oxygen, as depicted in panel A. In analyzing these data, our goal is to precisely measure the rates of inhibition and reactivation of the enzyme. Unlike in the experiment described in Figure 1, the only reason we repeat the injections in Figure 4 is to increase the statistics of the measurement.

In a recent paper about $C$. acetobutylicum $\mathrm{FeFe}$ hydrogenase, we showed that transients like those in Figure 4B could be fitted

(32) Baffert, C.; Demuez, M.; Cournac, L.; Burlat, B.; Guigliarelli, B.; Soucaille, P.; Bertrand, P.; Girbal, L.; Léger, C. Angew. Chem., Int. Ed. 2008, 47, 2052-2054, DOI: 10.1002/anie.200704313.

(33) Ludwig, M.; Cracknell, J. A.; Vincent, K. A.; Armstrong, F. A.; Lenz, O. J. Biol. Chem. 2009, 284, 465-477, DOI: 10.1074/jbc.M803676200.

(34) de Lacey, A. L.; Fernandez, V. M.; Rousset, M.; Cammack, R. Chem. Rev. 2007, 107, 4304, DOI: 10.1021/cr0501947.

(35) Jones, A. K.; Lamle, S. E.; Pershad, H. R.; Vincent, K. A.; Albracht, S. P. J.; Armstrong, F. A. I. Am. Chem. Soc. 2003, 125, 8505, DOI: 10.1021/ ja035296y.

(36) Parkin, A.; Cavazza, C.; Fontecilla-Camps, J.; Armstrong, F. J. Am. Chem. Soc. 2006, 128, 16808, DOI: 10.1021/ja064425i. 


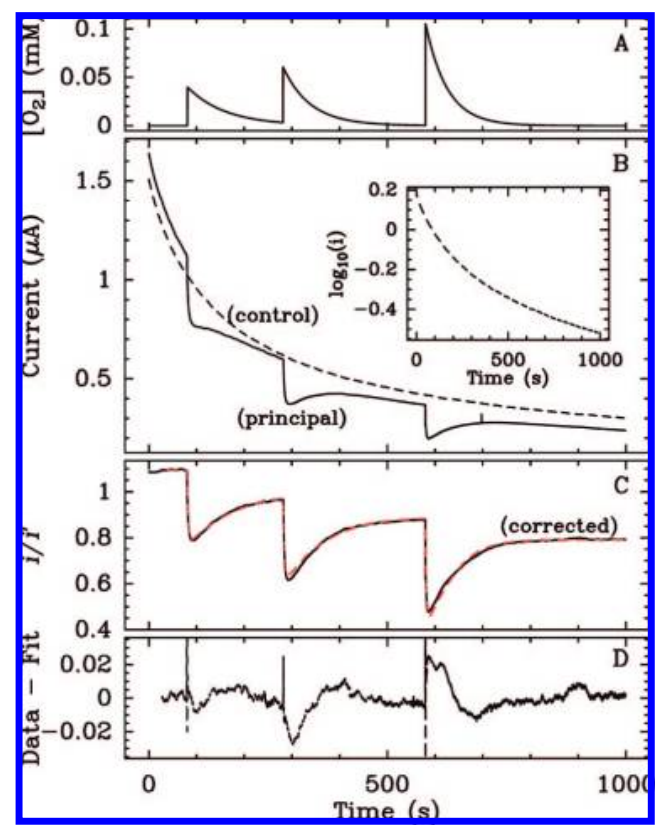

Figure 4. (A) Plot of $\mathrm{O}_{2}$ concentration against time, calculated using the volume of oxygenated buffer injected in the cell and the time constant of the decay deduced from fitting the data in panel B. (B) The black line shows how $C$. acetobutylicum FeFe hydrogenase is inhibited by successively injecting three aliquots of a buffer equilibrated under 1 atm of $\mathrm{O}_{2}$ (ref 32). Oxygen is flushed away by the stream of $\mathrm{H}_{2}$ and vanishes with a time constant of about $1 \mathrm{~min}$. The volumes of the aliquots divided by the total volume of buffer in the cell were $x=0.032,0.046$, and $0.085 ; \mathrm{pH} 7,25^{\circ} \mathrm{C}, \omega=2$ $\mathrm{krpm}, E=+190 \mathrm{mV}, 1 \mathrm{~atm}$ of $\mathrm{H}_{2}$. The dashed line shows the control signal recorded in an experiment carried out with a fresh film of enzyme handled in the same manner, except that no oxygen was added. (C) The plain line is the corrected signal obtained by dividing the principal signal in panel $\mathrm{B}$ by the control signal. This removes the effect of both film loss and anaerobic inactivation. The dashed line shows the fit of these data to the current equation given by Scheme 1B. Panel D shows the difference between the corrected data and the fit.

\section{Scheme 1. Schemes Used for Modelling the Inhibition of C. acetobutylicum FeFe Hydrogenase by $\mathrm{O}_{2}$}

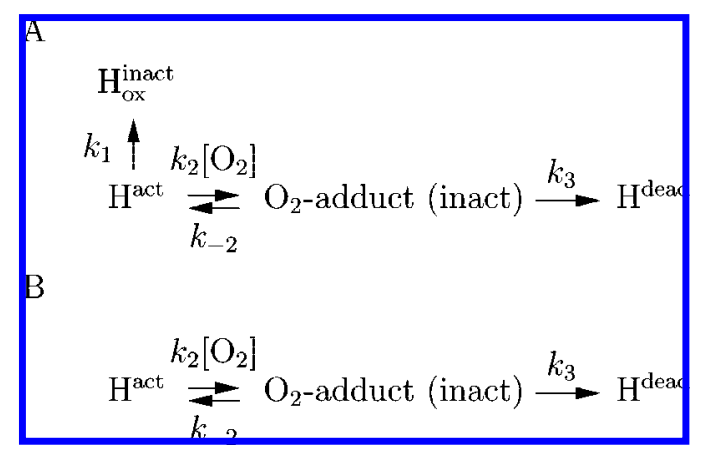

by using the model depicted in Scheme 1A, which assumes that the enzyme inactivates in two concurrent processes: the anaerobic formation of the $\mathrm{H}_{\text {inact }}^{\text {ox }}$ state, with a first-order rate constant $k_{1}$, and the two-step formation of an oxygen-damaged species (oxygen binds with a bimolecular rate constant $k_{2}$ to form an adduct which either dissociates with first-order rate constant $k_{-2}$ or irreversibly inactivates with rate $k_{3}$ ). The values of $k_{1}$ that were obtained by fitting the successive transients showed a twofold variability, as observed in the right panel of Figure 5 in ref 32. This results from the fact that when Scheme $1 \mathrm{~A}$ is used to fit the principal signal, the value of $k_{1}$ incorporates processes (film loss and anaerobic inactivation) that are approximated by a first-order reaction, whereas the inset in Figure 4B clearly shows that this approximation is wrong.

However, analyzing corrected data will prove more satisfactory. The corrected signal in Figure 4C (plain line), which results from dividing the principal signal in panel B by the control obtained under anaerobic conditions, is shown as a dashed line. The downward trend has disappeared, showing that the division has suppressed the effects of both film loss and anaerobic inactivation. That the corrected current reaches well-defined plateaus after each injection of oxygen demonstrates that the correction is trustful. The corrected signal is easily interpreted in terms of the simplified model depicted in Scheme 1B: the enzyme inactivates transiently when oxygen is injected, and the activity is mostly recovered after $\mathrm{O}_{2}$ has been flushed away; the fraction of activity that is not recovered corresponds to the enzyme that has irreversibly reacted with $\mathrm{O}_{2}$ (rate constant $k_{3}$ ). We no longer include anaerobic inactivation, which was represented by $k_{1}$ in Scheme $1 \mathrm{~A}$; therefore, the data are fitted by adjusting one fewer parameters. The dashed line in panel B was calculated by adjusting a unique set of rate constants: $k_{2}=3.1 \mathrm{~s}^{-1} / \operatorname{atm}\left(\mathrm{O}_{2}\right), k_{-2}=0.26$ $\mathrm{s}^{-1}, k_{3}=3.6 \times 10^{-3} \mathrm{~s}^{-1}$. The values obtained in ref 32 were similar, but the present analysis is more reliable because we no longer face the confusing observation that the apparent value of $k_{1}$ depends on time. Hence, this method provides a simpler and more accurate strategy than that used in ref 32 for quantitatively examining the reaction of the enzyme with oxygen. The correction is also greatly beneficial for interpreting the data in a qualitative manner: unlike the raw data, the corrected signal in panel B is a very clear footprint of how transient exposure to oxygen affects catalytic activity.

The above correction is particularly simple and efficient because the background decay in current that results from film loss and inactivation is very reproducible.

Synthetic Control Signal Interpolated or Extrapolated from Certain Parts of the Principal Signal. Last, we illustrate situations in which a control signal must be synthesized when the strategy consisting in dividing the principal experiment by a chronoamperogram obtained with a different film of enzyme fails. For this, we consider the same experiment as above, but carried out with mutants of the NiFe hydrogenase from Desulfovibrio fructosovorans $^{5,37}$ (solid lines in Figure 5A). Initially, we observed that proceeding as for FeFe hydrogenase returned "corrected" signals that did not show the expected plateaus, not even during the period preceding the injection of oxygen (see Supporting Information Figure S2). We concluded that the background decay in current was not reproducible enough that independent experiments could be used as controls.

If the effect of $\mathrm{O}_{2}$ were reversible on the time scale of the experiment, a control signal could be calculated by fitting a spline function to the current measured under anaerobic

(37) Dementin, S.; Leroux, F.; Cournac, L.; de Lacey, A. L.; Volbeda, A.; Léger, C.; Burlat, B.; Martinez, N.; Champ, S.; Martin, L.; Sanganas, O.; Haumann, M.; Fernández, V. M.; Guigliarelli, B.; Fontecilla-Camps, J. C.; Rousset, M. Submitted to J. Am. Chem. Soc. 


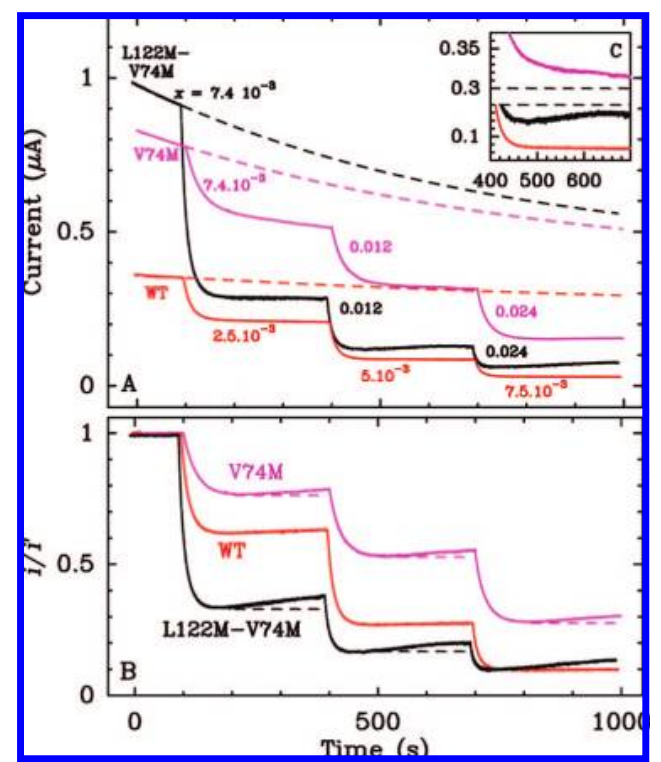

Figure 5. (A) Chronoamperograms showing the effect of oxygen on the activity of $D$. fructosovorans $\mathrm{NiFe}$ hydrogenase. The red signal was obtained with the wild type, the black with the L122M-V74M mutant, and the magenta with the $\mathrm{V} 74 \mathrm{M}$ mutant; $\mathrm{pH} 7,40^{\circ} \mathrm{C}, \omega=2$ $\mathrm{krpm}, E=+190 \mathrm{mV}, 1 \mathrm{~atm}$ of $\mathrm{H}_{2}$. The parameter $x$ denotes the volumic fractions of buffer equilibrated in air and injected in the cell solution. The dashed lines show the synthetic control signals constructed as explained in the text. Panel B shows the corrected data. The dashed lines are horizontal guidelines.

conditions (that is, before the injection of oxygen and after oxygen has vanished), and the data could be divided by this interpolated polynomial function. This is the strategy we used to correct for film loss the chronoamperograms for $\mathrm{CO}$ inhibition of nitrite reductase (see supplementary Figure S4 in ref 4) and NiFe hydrogenase, ${ }^{5}$ but it cannot be applied to the data in Figure 5A, since in this case oxygen inhibition is mostly irreversible.

To design a strategy for correcting the data in Figure 5A, we characterized a number of signals recorded while NiFe hydrogenase was inactivating at high electrode potential in the absence of oxygen, and we found that the chronoamperograms could always be fitted to $y(t)=a \exp (-t / \tau)+b$. Moreover, analyzing several chronoamperograms recorded with the same film of enzyme, the value of $\tau$ was reproducible (within 15\%) and of the order of the characteristic time of the experiment (about $1000 \mathrm{~s}$ ), see Supporting Information Figure S3. This allowed us to calculate the synthetic control signals shown as dashed lines in Figure 5A in the following manner: (i) we recorded chronoamperograms showing the inactivation of the enzyme in the absence of oxygen and we fitted them to determine the average time constant $\tau_{\mathrm{av}}$; (ii) we fitted the quasi-linear phase of the principal signal $(0<$ $t<100 \mathrm{~s}$ in Figure 5A) to $y(t)=a \exp \left(-t / \tau_{\mathrm{av}}\right)+b$; (iii) we used this function to extrapolate the control signal over the entire time frame of the experiment. (Supporting Information Figure S3 illustrates the complete series of anaerobic and aerobic experiments carried out to record these signals.)

Importantly, the above method is unbiased: it makes no assumption about whether or not the inhibition is reversible since the current recorded after exposure to $\mathrm{O}_{2}$ is not used to calculate the control signal.
Generally speaking, the simpler strategy which would have consisted in fitting the initial, anaerobic part of the data to $y(t)=$ $a \exp (-t / \tau)+b$ by adjusting three parameters (rather than two above) is unlikely to predict a correct control function. Indeed, if the time constant of the decay is of the order of, or larger than, the time scale of the experiment, it cannot be deduced from the anaerobic, linear region of the data, and the three parameters are ill-defined. Alternatively, if $\tau$ is small and $b / a$ is small, that is, the current rapidly decays to zero, the extrapolated function and the corrected data will be very sensitive to the value of $\tau$. Last, if $\tau$ is small and $b / a$ is not small, it would make more sense to wait until the initial decay phase is over before proceeding with oxygen injections, and to use uncorrected data.

Figure $5 \mathrm{~A}$ shows the chronoamperograms recorded with wildtype (WT) NiFe hydrogenase (in red), and the L122M-V74M and V74M mutants ${ }^{5,37}$ (black and magenta, respectively). For the WT and V74M mutants, the activity continuously decreases as a consequence of film loss and both anaerobic and aerobic inactivation; the latter slows each time oxygen is flushed away by the stream of $\mathrm{H}_{2}$. The L122M-V74M mutant behaves differently: there is a small amplitude reactivation after oxygen has been removed from the cell (see the inset). This partial recovery of activity is significant because under the very oxidizing conditions used in this experiments, one expects nothing but the inactivation of the enzyme. We wondered whether this reactivation process also takes place in the V74M and WT enzymes, but at a slower rate, so that it is overcome by film loss and anaerobic inactivation. The corrected data shown in panel B provide the answer: a small but meaningful reactivation phase is apparent for the $\mathrm{V} 74 \mathrm{M}$ enzyme once the data have been corrected for film loss and anaerobic inactivation, whereas the corrected current given by the WT enzyme reaches welldefined plateaus before and after each injection of oxygen.

We conclude that in the V74M mutant, but not in the WT, the reactivation observed for the L122M-V74M mutant can occur and yet be undetected because it is slow and it can easily be blurred by film loss and anaerobic inactivation. The spectacular observation here is that correcting the data uncovers an intriguing feature that is hidden in the raw signal.

\section{CONCLUSION}

In many different fields, and indeed in spectrometry, chromatography, and electrochemistry alike, correcting data usually involves subtracting a background signal. This is so because the different elements which contribute to a particular result are generally additive. For example, in electrochemistry, the capacitive current always adds to the faradaic signal, and the currents that arise from independent reactions can be deconvolved because they are cumulative also.

In contrast, we have shown in this paper that in various situations the contributions to a chronoamperogram are multiplicative, and correcting the data to take away the influence of a certain contribution must involve a division rather than a subtraction. That only the latter operation is available in commercial electrochemistry softwares suggests that this conclusion had not been grasped before.

We exposed a method that makes it possible to "remove" from a chronoamperogram the influence of processes that cannot be eliminated from an experiment defined as the control experiment. 
The delicate point of the method is to obtain a reliable control signal. We have shown that it can be synthesized in the most common situation in which the variation of turnover rate consists of discrete steps due to the stepwise addition of substrate or inhibitors. When the time dependence of the catalytic rate is more complex, a control signal must be recorded, which is such that the variation of electroactive coverage is the same as (or very close to that) in the principal experiment. This requires that the process whose kinetics one seeks to define does not interfere significantly with the background decrease in current. Different strategies can be used for obtaining the control signal. It may be possible to record it with a different film handled as in the principal experiment or from a subsequent experiment carried out with the same film. Alternatively, one may use a spline function to interpolate the control signal, ${ }^{4}$ or model film loss or inactivation by fitting several control experiments to an ad hoc function, and use the result as a control signal.

\section{METHODS}

The data were analyzed using an inhouse program called SOAS, which is available free (under GNU General Public License) ${ }^{38}$ and free of charge on our Web site. ${ }^{39}$ Mac OS X binaries can also be downloaded, thanks to Dr. Kevin Hoke (Department of Chemistry, Berry College, Mount Berry, GA). This program proposes commands for handling voltammetric and chronoamperometric data, including smoothing signals, differentiation, subtracting baselines, fitting current responses, measuring limiting currents, and searching for peak positions. Dividing two data sets is obtained with the "div" command. Dividing a signal by an ad hoc (user-defined) baseline is obtained with the "v" key while the baseline is being adjusted (a number of options are available for defining baselines). Analyzing chronoamperograms according to the procedure described in Figures 1 and 2 is simplified by the film_decay command, as described in the online manual, ${ }^{40}$ under the heading "baseline corrections."

The samples of $R$. sphaeroides nitrate reductase ${ }^{26,41}$ and $D$. fructosovorans NiFe hydrogenase mutants were prepared as described previously. ${ }^{5,42,43}$ Homologous expression of $C$. acetobutylicum $\mathrm{FeFe}$ hydrogenase HydA1 in the form of a C-terminal Strep-tag-II-tagged protein was performed using $C$. acetobutylicum (pPHhydACa-C-tag) strain using the procedure of Girbal et al. ${ }^{44}$ modified by Demuez et al. ${ }^{45}$ To improve purification yield and

(38) GNU General Public License, version 2.0 GNU Project-Free Software Foundation (FSF), http://www.gnu.org/licenses/old-licenses/gpl-2.0.html (accessed March 1, 2009).

(39) SOAS Electrochemistry software, http://bip.cnrs-mrs.fr/bip06/software.html. Fourmond, V.; Hoke, K.; Heering, H. A.; Baffert, C.; Leroux, F.; Bertrand, P.; Léger, C. Bioelectrochemistry, in press, 2009, DOI: 10.1016/ j.bioelechem.2009.02.010.

(40) SOAS Manual, http://bip.cnrs-mrs.fr/bip06/soas/. purified hydrogenase concentration, the protocol was further modified as follows. To limit oxygen exposure, the entire purification is performed in an anaerobic glovebox (JACOMEX); this includes cells recovery, centrifugation, and proteins extraction. To speed up the purification, the one-step affinity chromatography is done with a $5 \mathrm{~mL}$ Strep-Tactin Superflow (IBA, Göttingen, Germany) column connected to an Äkta purifier 10 system located in the anaerobic glovebox.

The electrochemical cell, thermostatted using a water circulation, was housed in a Faraday cage. A pyrolytic graphite edge rotating disk working electrode (area $\approx 5 \mathrm{~mm}^{2}$ ) was used in conjunction with an EG\&G M636 electrode rotator, a platinum wire was used as a counter electrode, and a saturated calomel electrode (SCE), located in a Luggin side arm containing $0.1 \mathrm{M} \mathrm{NaCl}$ and maintained at room temperature, was used as a reference. All potentials are quoted versus the standard hydrogen electrode (SHE), using $E_{\mathrm{SHE}}=E_{\mathrm{SCE}}+241 \mathrm{mV}$. Experiments were performed with an Autolab electrochemical analyzer (PGSTAT 12, Eco Chemie) and carried out in an anaerobic glovebox (JACOMEX) filled with $\mathrm{N}_{2}$ (residual $\mathrm{O}_{2}$ $<1 \mathrm{ppm}$ ). The methods we use to form the enzyme films were described previously. ${ }^{29,32,43}$ The buffer contained $5 \mathrm{mM}$ each of MES, sodium acetate, HEPES, TAPS, and CHES, $0.1 \mathrm{mM}$ EDTA, and $0.1 \mathrm{M} \mathrm{NaCl}$.

\section{ACKNOWLEDGMENT}

Our work is funded by the CNRS, CEA, INRA, INSA, ANR, Aix-Marseille Université, and the city of Marseilles. We acknowledge support from the "pôle de compétitivités CAPENERGIES".

\section{SUPPORTING INFORMATION AVAILABLE}

Supplementary Figures S1-S3. This material is available free of charge via the Internet at http://pubs.acs.org.

Received for review December 5, 2008. Accepted February 18, 2009.

\section{AC8025702}

(41) Dementin, S.; Arnoux, P.; Frangioni, B.; Grosse, S.; Léger, C.; Burlat, B.; Guigliarelli, B.; Sabaty, M.; Pignol, D. Biochemistry 2007, 46, 9713, DOI: 10.1021/bi700928m.

(42) Dementin, S.; Burlat, B.; de Lacey, A. L.; Pardo, A.; Adryanczyk-Perrier, G.; Guigliarelli, B.; Fernandez, V. M.; Rousset, M. L. Biol. Chem. 2004, 279, 10508, DOI: 10.1074/jbc.M312716200.

(43) Dementin, S.; Belle, V.; Bertrand, P.; Guigliarelli, B.; Adryanczyk-Perrier, G.; Delacey, A.; Fernandez, V. M.; Rousset, M.; Léger, C. I. Am. Chem. Soc. 2006, 128, 5209, DOI: 10.1021/ja060233b.

(44) Girbal, L.; von Abendroth, G.; Winkler, M.; Benton, P. M.; Meynial-Salles, I.; Croux, C.; Peters, J. W.; Happe, T.; Soucaille, P. Appl. Environ. Microbiol. 2005, 71, 2777-2781, DOI: 10.1128/AEM.71.5.2777-2781.2005.

(45) Demuez, M.; Cournac, L.; Guerrini, O.; Soucaille, P.; Girbal, L. FEMS Microbiol. Lett. 2007, 275, 113-121, DOI: 10.1111/j.1574-6968.2007.00868.x. 